\title{
An Analysis of Sleep and Ergometer Performance in Collegiate Male Rowers
}

THADDEUS BABIEC, ${ }^{1}$ DANIEL ROSNEY, $\mathrm{PhD}^{1}$

${ }^{1}$ Department of Kinesiology, Temple University College of Public Health

Correspondence: tclbabiec@gmail.com (Thaddeus Babiec)

Introduction: Research has increasingly examined the effects of sleep on atbletic performance. Although there is substantial evidence for the detrimental effects of sleep deprivation on athletic performance, few studies have assessed the effects of sleep extension, and most are limited to field or team sports. Additionally, existing sleep extension studies largely exclude sports such as rowing which traditionally have early practice times and therefore represents a population at heightened risk of sleep deprivation. The purpose of the present study is to determine whether sport specific performance benefits are gained when collegiate rowers extend their sleep. Methods: Nineteen members of the Temple University men's rowing team were asked to increase their sleep to nine to ten hours (540-600 minutes) a night for four weeks, following a two-week baseline period. A two-week postintervention phase followed the sleep extension period. In addition to daily heart rate variability, three sport-specific measures - related to peak rowing power and pace - were obtained weekly using a rowing ergometer. Results: Subjects were unable to extend their sleep from baseline during the intervention phase, $392.07 \pm 53.69$ minutes and $374.11 \pm 41.53$ minutes, respectively $(p=.137)$. Significant variation was found in the week to week comparison of the Interval test and OR1-Min test. Conclusion: Since athletes failed to increase their sleep time, we were unable to examine the impact of sleep on performance. It is likely that athletes require more education or access to other strategies to develop the habits necessary for increased sleep. Changes in performance from baseline could be attributed to unmeasured variables, such as stress, nutrition, or changing workload in training.

\section{Introduction}

Sleep deprivation and restriction on athletic performance has been central in sleep literature focused on athletics for the past few decades. Studies have questioned the effects of sleep deprivation on athletes and performance in varying age groups, sports, and ability levels. Studies have consistently reported findings of detriment to performance in endurance events, such as time to exhaustion and time trial events, and in power sports such as powerlifting and sprinting. Similar results have been found across the spectrum of talent when athletes are subjected to mild to severe sleep deprivation. ${ }^{1-6}$ Conversely, few studies explore the effects of sleep extension and increased total sleep time on athletic performance. Widely cited research out of Stanford University found significant improvements in sport specific skills, mood, and hand-eye coordination following and during an eight-week sleep extension study with collegiate basketball players. ${ }^{7}$ Since that publication, several other studies have found similar results in sports such as rugby, soccer, and tennis to those in the Stanford study. ${ }^{8,}$

Athlete monitoring and biofeedback has provided a new, technology driven method for training high level athletes. Heart rate variability (HRV), a measure of the change in time between consecutive heartbeats, or the R-R interval, has been shown to be an accurate predictor of recovery. ${ }^{10} \mathrm{HRV}$ is a 
reflection of vagal nerve activation which controls parasympathetic and sympathetic nervous system activity. ${ }^{11}$ Higher HRV values generally correlate with greater health and recovery while lower values with worse recovery and greater risk of injury and illness. ${ }^{12-14}$ As sleep is the primary time when mental and physical recovery occurs, daily HRV recordings provide an objective measure of the recovery quality from the previous night of sleep.

The ability of an athlete to recover will directly influence how well they will perform on consecutive days of practice and competition. ${ }^{15,16}$ Effectively monitoring how long it takes an athlete to recover from a training session or competition can give insights into the training strategies being used and what modifications may be needed. ${ }^{17}$ Utilizing HRV as a method of monitoring fatigue and "readiness" in athletes and teams correlates with performance and injury risk in a host of different athletes, suggesting a simple and cost effective tool for coaches. ${ }^{17-19}$

Rowing is a sport with unique physical requirements, with athletes requiring both high levels of endurance as well as strength. As a sport with traditionally early practice and race times, athletes often do not obtain the recommended seven to nine hours of sleep each night. ${ }^{20}$ With sleep deprivation a known hindrance to performance, observing these athletes obtaining eight or more hours of sleep each night will provide continued insight into both the effects of sleep deprivation, as well as the effects of recommended and extended amounts of sleep. As an underserved and physiologically different group of athletes, rowers provide a unique population for expanded work on sleep extension.

This study aims to determine whether a period of sleep extension leads to improvement on ergometer performance in collegiate male rowing athletes. We hypothesize that there will be improvement on the rowing ergometer performance as a result of sleep extension and that HRV will increase as sleep increases.

\section{Methods}

Nineteen rowers from the Temple Men's Rowing team elected to participate in this study. To assess natural changes in sleep, all participants were to refrain from any caffeine consumption and to avoid the use of diphenhydramine containing sleep aids such as ZzzQuilC or supplements such as melatonin. Any participants who were taking prescribed or over-the-counter medication that affected sleep were excluded from this study. Alcohol consumption was prohibited. Participants were excluded if they had any sleep disorders. Screening was done using three validated questionnaires to assess daytime sleepiness, sleep quality, and sleep apnea risk; the Epworth Sleepiness Scale, the Pittsburgh Sleep Quality Index, and the STOP-Bang. ${ }^{21-23}$

Prior to participation, all subjects were given material pertaining to the specific aims and goals of the study and all subjects provided written informed consent. Ethical approval for this study was given by the Temple University Institutional Review Board.

This eight-week study employed a three phase within-subjects study design (Figure 1). Data collection began at the start of the 2019 spring semester with a two-week (13 night) baseline period. At the start of the third week, participants were instructed to increase their sleep to a minimum of nine hours each night for four weeks. If subjects were unable to achieve this amount of sleep each night, they were instructed to make up the rest of the time through naps during the day. Following the four-week sleep extension intervention, subjects returned to baseline levels of sleep for another two-week period. 
Figure 1. Study Timeline

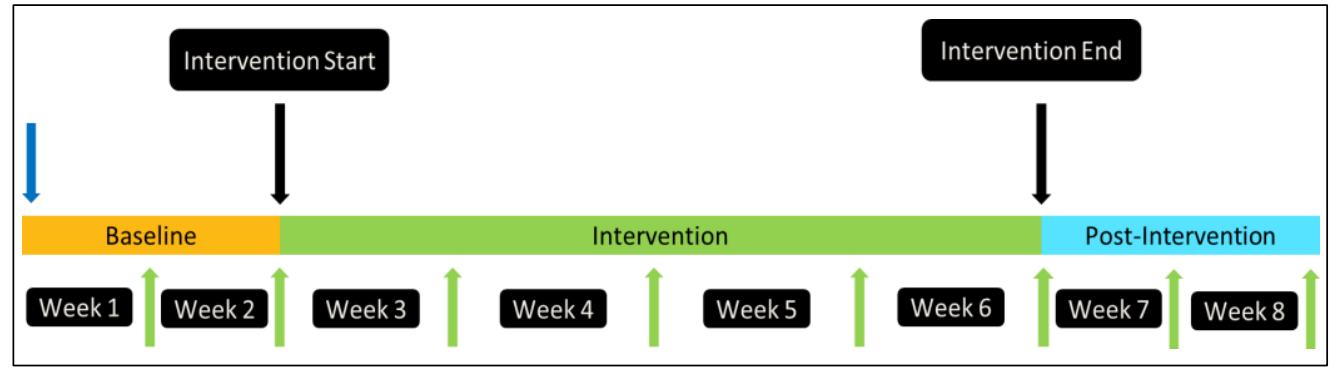

Note: Blue arrow represents Informed Consent and administration of screening questionnaires. Green arrows indicate data collection and compilation for the proceeding week. Baseline consisted of Weeks 1 and 2, Intervention Weeks 3 through 6, and PostIntervention consisted of Weeks 7 and 8. Each week, sport specific measures of performance, daily HRV, and total sleep were recorded and given a weekly average for each participant. Each participant performed and reported two weekly sport specific measures of performance (Table 1).

Table 1. Outcome and Sleep Measures at Weekly Intervals

\begin{tabular}{|c|c|c|c|c|c|c|c|c|c|c|c|c|c|c|c|c|c|c|c|c|c|c|c|c|}
\hline & \multicolumn{3}{|c|}{ Week 1} & \multicolumn{3}{|c|}{ Week 2} & \multicolumn{3}{|c|}{ Week 3} & \multicolumn{3}{|c|}{ Week 4} & \multicolumn{3}{|c|}{ Week 5} & \multicolumn{3}{|c|}{ Week 6} & \multicolumn{3}{|c|}{ Week 7} & \multicolumn{3}{|c|}{ Week 8} \\
\hline & $\mathrm{N}$ & Avg & $\mathrm{SD}$ & $\mathrm{N}$ & Avg & SD & $\mathrm{N}$ & Avg & SD & $\mathrm{N}$ & Avg & SD & $\mathrm{N}$ & Avg & SD & $\mathrm{N}$ & Avg & $\mathrm{SD}$ & $\mathrm{N}$ & Avg & SD & $\mathrm{N}$ & Avg & SD \\
\hline OR1-Min (W) & 0 & & & 10 & 798.8 & 79.87 & 4 & 718.5 & 70.56 & 10 & 774.2 & 101.49 & 5 & 781.8 & 92.39 & 8 & 782.63 & 132.83 & 9 & 749.89 & 143.14 & 5 & 727 & 144.71 \\
\hline RC1-Min (W) & 0 & & & 13 & 470.62 & 47.95 & 13 & 461.23 & 53.96 & 13 & \begin{tabular}{|l|}
459.69 \\
\end{tabular} & 49.68 & 11 & 464.27 & 42.03 & 11 & 466.09 & 60.63 & 12 & 466.42 & 51.48 & 9 & 471.33 & 48.98 \\
\hline Interval Test (W) & 0 & & & 11 & 444.45 & 36.1 & 12 & 415.5 & 59.25 & 13 & 402.23 & 42.42 & 10 & 435 & 29.81 & 8 & 419.63 & 37.18 & 10 & 432.3 & 34.73 & 9 & 442 & 32.62 \\
\hline $\mathrm{HRV}(\mathrm{ms})$ & 12 & 119.26 & 50.05 & 11 & 111.92 & 53.51 & 13 & 118.68 & 58.62 & 13 & 133.74 & 78.24 & 12 & 152.64 & 83.1 & 11 & 125.54 & 71.48 & 11 & 131.02 & 56.78 & 9 & 117.5 & 61.6 \\
\hline TST (min) & 11 & 408.52 & 60.53 & 13 & 385.65 & 53.7 & 13 & 383.91 & 45.45 & 12 & 387.84 & 50.6 & 12 & 371.06 & 38.78 & 8 & 370.13 & 36.34 & 9 & 372.24 & 31.58 & 10 & 364.1 & 41.49 \\
\hline $\mathrm{TIB}(\min )$ & 11 & 480.65 & 52.28 & 13 & 477.53 & 51.93 & 13 & 450.08 & 128.13 & 12 & 482.69 & 65.63 & 12 & 475.47 & 57.94 & 8 & 496.77 & 45.23 & 9 & 465.19 & 26.49 & 10 & 447.17 & 34.41 \\
\hline
\end{tabular}

Note: OR1-Min = Open Rate 1 Minute Test; RC1-Min = Rate Capped 1 Minute Test; HRV = Heart Rate Variability; TST $=$ Total Sleep Time; TIB = Time in Bed. All significant changes are highlighted in yellow. Significant changes in ORI-Min were found between Weeks 2 and 3, and Weeks 3 and 5 ( $p<$ 0.05). Significant changes in the Interval Test were found between Weeks 4 and $8(p<0.05)$.

\section{Sport Specific Tests}

Measurements of rowing performance were collected weekly through an Open-rate 1-Minute test (OR1Min), a Rate-capped 1-Minute Test (RC1-Min) and an Interval Test (Interval). The two 1-minute tests are variations on peak power testing common in the rowing community. The Interval test is a modified version of a 2000-meter predictor workout, 6x500m intervals. All three tests were on a Concept 2 Rowing Ergometer (Concept2, Morrisville, VT). The OR1-Min was a maximal 1-minute effort. No guidance was given to pacing. Total output was measured in average wattage.

The RC1-Min was a maximal effort one-minute test at a controlled stroke rate or rhythm of 30 strokes per minute (spm). Total output was recorded in average wattage. For the Interval Test, participants were directed to perform three maximal effort 90 second intervals with two minutes rest between each. There was no designated stroke rate for this test. The average wattage over the three intervals was recorded. To minimize confounding factors, testing was prescribed for a specific day of the week; Wednesday for the OR1-Min and RC1-Min and Friday for the Interval test. Participants could select what time of day they wished to complete the testing to accommodate class schedules. 
Baseline testing for OR1-Min, RC1-Min, and Interval tests were performed during Week 2. All data points are represented as the average value of each study week, with subjects who reported $75 \%$ or greater of their data each week included in analysis. Measurements of rowing performance were collected weekly through an Open-rate 1-Minute test (OR1-Min), a Rate-capped 1-Minute Test (RC1-Min) and an Interval Test (Interval). The two 1-minute tests are variations on peak power testing common in the rowing community. The Interval test is a modified version of a 2000 -meter predictor workout, $6 \times 500 \mathrm{~m}$ intervals. All three tests were on a Concept 2 Rowing Ergometer (Concept2, Morrisville, VT). The OR1-Min was a maximal 1-minute effort. No guidance was given to pacing. Total output was measured in average wattage.

The RC1-Min was a maximal effort one-minute test at a controlled stroke rate or rhythm of 30 strokes per minute (spm). Total output was recorded in average wattage. For the Interval Test, participants were directed to perform three maximal effort 90 second intervals with two minutes rest between each. There was no designated stroke rate for this test. The average wattage over the three intervals was recorded. To minimize confounding factors, testing was prescribed for a specific day of the week; Wednesday for the OR1-Min and RC1-Min and Friday for the Interval test. Participants could select what time of day they wished to complete the testing to accommodate class schedules. Baseline testing for OR1-Min, RC1-Min, and Interval tests were performed during Week 2. All data points are represented as the average value of each study week, with subjects who reported $75 \%$ or greater of their data each week included in analysis.

Measurements of rowing performance were collected weekly through an Open-rate 1-Minute test (OR1Min), a Rate-capped 1-Minute Test (RC1-Min) and an Interval Test (Interval). The two 1-minute tests are variations on peak power testing common in the rowing community. The Interval test is a modified version of a 2000-meter predictor workout, 6x500m intervals. All three tests were on a Concept 2 Rowing Ergometer (Concept2, Morrisville, VT). The OR1-Min was a maximal 1-minute effort. No guidance was given to pacing. Total output was measured in average wattage.

The RC1-Min was a maximal effort one-minute test at a controlled stroke rate or rhythm of 30 strokes per minute (spm). Total output was recorded in average wattage. For the Interval Test, participants were directed to perform three maximal effort 90 second intervals with two minutes rest between each. There was no designated stroke rate for this test. The average wattage over the three intervals was recorded. To minimize confounding factors, testing was prescribed for a specific day of the week; Wednesday for the OR1-Min and RC1-Min and Friday for the Interval test. Participants could select what time of day they wished to complete the testing to accommodate class schedules.

Baseline testing for OR1-Min, RC1-Min, and Interval tests were performed during Week 2. All data points are represented as the average value of each study week, with subjects who reported $75 \%$ or greater of their data each week included in analysis.

Sleep recordings were collected by the subjects on a nightly basis using a SleepScore Max device (SleepScore Labs, Carlsbad, CA). The SleepScore Max uses ultra-wide band, comparable to ultra-low energy radar, to track the movement and respiration of an individual in bed. ${ }^{24} \mathrm{~A}$ validated technology against both actigraphy and polysomnography. ${ }^{25}$ Data collection started when the subject turned the lights out and ended when they woke up the next morning. Total Sleep Time (TST) and Time in Bed (TIB), or the total length of a recording, were assessed on a daily and weekly basis. If subjects were unable to obtain 9 hours of sleep at night, they were encouraged to nap during the day for the remainder of the time. TST and TIB values for naps were collected using the SleepScore Max and added to the TST and TIB from the previous night for cumulative minutes. For analysis, participants who recorded four or fewer nights of sleep during a given week were excluded from the weekly analysis of that week. 


\section{Daily HRV Recording}

HRV data was collected each morning through the HRV4Training smartphone app, validated in several peer reviewed studies, using photoplethysmography (PPG). ${ }^{26-29}$ Following standard procedures, upon waking, the participants were to sit up comfortably in bed and take their morning HRV. Subjects were instructed to cover the camera lens and camera light of their smartphone device with their finger. The HRV4Training app software uses PPG to detect the changes in blood flow by illuminating the skin and measuring changes in light absorption. Once a strong PPG signal was established, subjects were instructed to sit still, breathing normally, for 60 seconds. Following the collection of the HRV measurement, participants were prompted to complete a short questionnaire of subjective measures of sleep, stress, fatigue, motivation, and lifestyle. Ratings were on a sliding Likert-type scale. If a subject failed to record a minimum of three HRV readings in a given week, their data was not considered statistically viable and was dropped from analysis for that week. ${ }^{30}$

Baseline values for HRV, TST and TIB are based on the average of Week 1 and Week 2 for each variable (Table 1). All data points are represented as the average value of participants who met the requirements listed above for minimum data collection (see Table 1).

\section{Statistical Analysis}

A power analysis run prior to data collection to determine appropriate sample sizes for the study concluded 18 participants were needed to have sufficient power for analysis. One-way ANOVA for repeated measures with a Bonferroni post-hoc analysis was run on the weekly averages of our outcome variables, OR1-Min, RC1-Min, Interval, and HRV to determine week to week variability in outcome measures. Statistical significance was set at $p<0.05$. Analyses and figures were performed and produced using SPSS software (Version 26, IBM Corp, New York, NY, USA) and Microsoft Excel 2017 (Redmond, WA, USA).

\section{Results}

Of the 19 initial participants, five (26\%) dropped out. Three due to an inability to follow the sleep extension protocol. One was lost to follow-up, and one was dropped in the fourth week of the study due to starting medication that affected sleep; their data was retained until Week 4. At study completion our $\mathrm{n}=14$, average age $=19.14 \pm .77 \mathrm{yrs}$, average height $=183.42 \pm 5.99 \mathrm{~cm}$, and average weight $=80.21 \pm$ $9.22 \mathrm{~kg}$.

The average results for each week of the study for OR1-Min, RC1-Min, HRV, TST, and TIB are reported in Table 1. There was no significant change between baseline and intervention TIB but there was a nonsignificant reduction in TST of 18 minutes during intervention (392.07 \pm 53.69 vs. $374.11 \pm 41.53, p=$ .137) (Figure 2). There was an increase in HRV during intervention but no significant differences between weeks (Figure 2).

Significant differences were found in the weekly OR1-Min results $(\mathrm{F}(1,6)=3.392, p<0.05)$, with pairwise comparison finding significant decrease between Week 2 and Week $3(\mathrm{M}=80.3 \mathrm{SD}=16.06, p<0.05)$, and a significant increase between Week 3 and Week $5(\mathrm{M}=-63.3, \mathrm{SD}=11.60, p<0.05)$ (Figure 3). Significant differences were also seen in the weekly Interval results $(\mathrm{F}(1,6)=4.555, p<0.05)$ with pairwise comparison indicating significant increase between Week 4 and Week $8(402.23 \pm 42.42,442.00 \pm 32.62$; $\mathrm{M}=-39.77, \mathrm{SD}=9.943, p<0.05)$ (Figure 3). 
Figure 2. HRV (rMSSD) and TST (Minutes) over the course of the study.

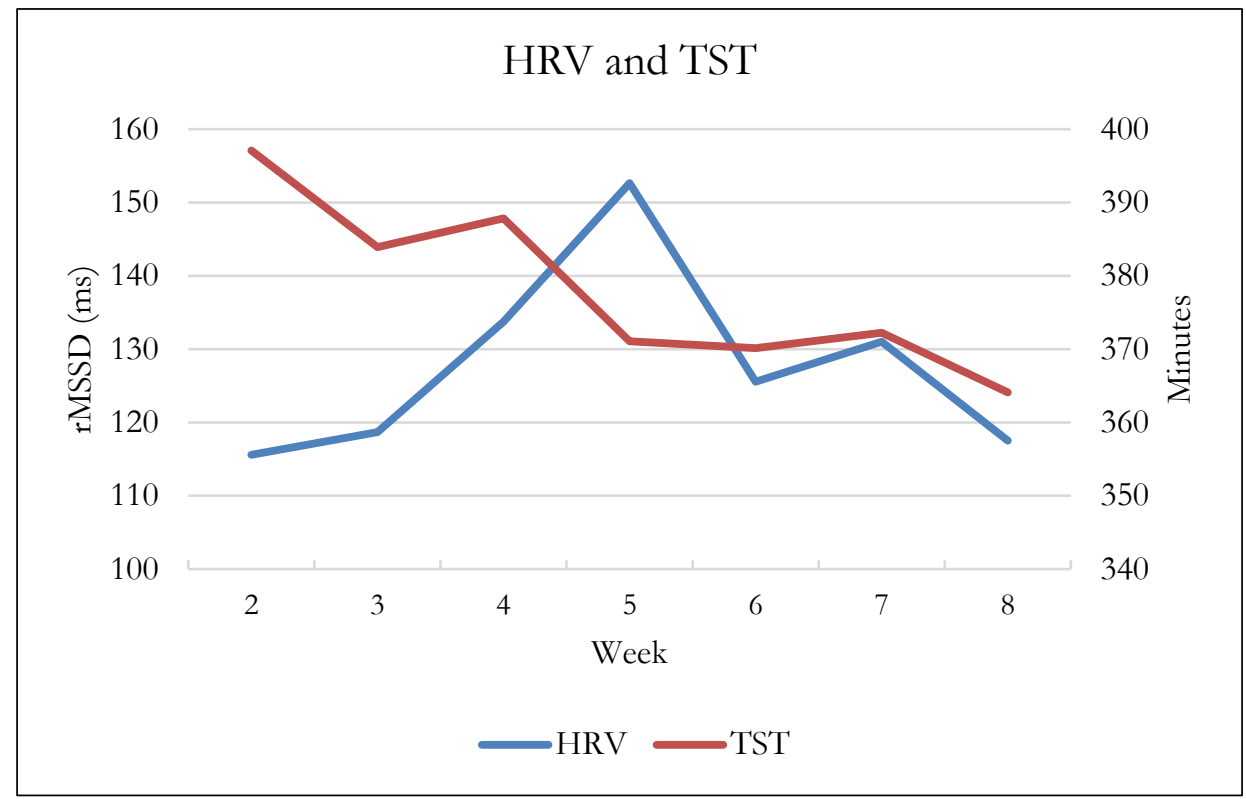

Note. Intervention phase from Week 3 through Week 6.

Figure 3. All performance tests during the study.

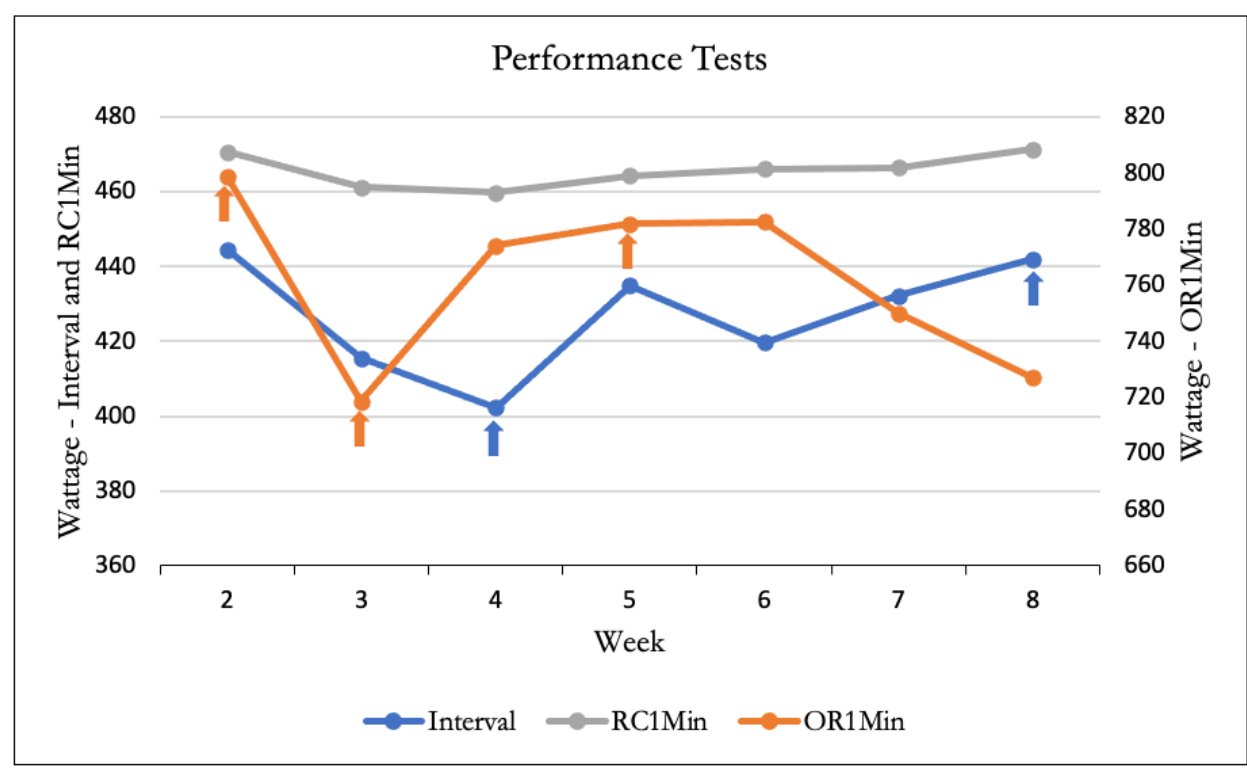

Note. Interval and RC1Min use the left y-axis, OR1Min uses the right y-axis. Significant week to week changes are indicated by arrows: orange arrows are for OR1Min, blue arrows are for Interval test. All significance at $p<0.05$. 


\section{Discussion}

We hypothesized that increased time asleep each night would lead to improvements in ergometer performance in these male rowers. Conclusions could not be interpreted due to the lack of sleep improvements during the intervention phase. We found no change in TIB between baseline and intervention phases but a decrease of 18 minutes in TST. TIB stayed consistent at approximately eight hours for the baseline and intervention phases of the study and dropped by approximately 20 minutes during post-intervention. Baseline and post-intervention TIB were expected to be similar, therefore this difference could suggest that the baseline measurements taken were not indicative of true habitual sleep patterns for participants. A probable cause is due to baseline collection occurring during the start of the school semester when participants could take advantage of the lighter workload to get increased amounts of sleep compared to what they would normally get during the semester, thus skewing the data. Conversely, it is possible that the post-intervention phase reflected a non-habitual sleep pattern, i.e., sleeping less than normal, allowing for the possibility that the pre- and intervention phases were indicative of habitual sleep patterns. Previous research has found average TST to be $416.0 \pm 22.2$ minutes in elite junior rowers (TIB $=446.1 \pm 16.3$ minutes) and $442 \pm 56$ minutes in elite male rowers. ${ }^{20,31}$ How these values relate to normal sleep times in collegiate male athletes remain unclear and future research should explore that question.

HRV behaved as anticipated based on prior research, increasing overtime to suggest a tolerance or adaptation to prescribed workload, though it did not correlate with sleep or performance. Previous research has established HRV as a proxy for recovery and adaptation to stress. ${ }^{10,18,28,32}$ Within our population, HRV increased during the fourth and fifth week of the study, indicating a tolerance and adaptation to the workload from the coaches. During the sixth week, when the team traveled and the training load increased, we observed a subsequent drop in HRV. Despite the behavior of the HRV data (see Figure 2), no correlation was found with sleep time. HRV is influenced by many different factors, sleep and training load are only a few of these. ${ }^{11,33-34}$ These other factors should be considered in future research.

There were significant changes in performance during the study in OR1-Min test and Interval test results. For the OR1-Min test, a significant decrease in performance was found between Week 2 and Week 3 of testing. Although it is unclear what the reasons for this might be, it possibly had something to do with the beginning of the sleep extension phase of the study. Prior research suggests improvements would be seen during and post sleep extension ${ }^{7,9,35-36}$ but, possibly transitioning from baseline to intervention phase could have triggered stress or other factors that negatively influenced the performance on the OR1-Min. The start of the spring semester may have played an additional role, with the change from a vacation to a school schedule and workload. This seems to be further supported since performance on the OR1-Min improved close to baseline levels by Week 5 (798.80 $\pm 70.05,781.80 \pm 57.30)$, suggesting participants had adjusted to their new schedule and the stress of a new semester was not present.

Only the Interval Test showed significant differences (Week 4 and Week 8). With any repeated test of performance, improvements in test results are expected as a function of practice and familiarization with the test. Only two outcome measures (OR1-Min and Interval) showed significant differences; possible explanations for this are discussed below.

There were no performance improvements from baseline to post-intervention. Performance across all measured outcome variables remained constant or got worse with performance declining in the OR1Min and the Interval tests between baseline post-intervention ( $798.80 \pm 78.38$ vs. $756.22 \pm 152.42 ; 444.45$ \pm 41.16 vs. $434.83 \pm 40.49)$. Since there were no significant changes made in either TST or TIB, if improvements in performance were observed, they would have been attributed expected changes due to 
daily practice. Even when controlling for weight and minimizing the influence of outliers, performance still declined (Data not shown). Though impossible to determine the influence sleep had on test performance, it is worth noting that with total sleep less than the amount recommended by the National Sleep Foundation (7-9 hours), performance decreased over time, suggesting the need for a minimum amount of sleep to expect performance improvements in rowing.

A common observation throughout this study and in previous research is the poor sleep behavior and hygiene of athletes. Both Mah and Swinbourne note that their data support previous findings characterizing the poor normative sleep behavior in athletes. ${ }^{7,9}$ Poor sleep behaviors can be the result of many factors. Lack of education seems to play a role, which includes knowledge about the importance of a consistent bedtime and individual sleep requirements. Individual requirements for sleep will change depending on the lifestyle demands of the athlete and inconsistent sleep schedules can negatively affect sleep and performance. ${ }^{37-38}$ Education programs around individual sleep requirements and developing consistent sleep schedules have shown to be effective in producing positive effects on these indices of sleep in both men and women. ${ }^{37,39}$ Additional factors to consider when assessing the poor sleep behavior of athletes are length and timing of sleep extension interventions, training load, and intensity. ${ }^{9}$ Sleep times decreased during the course of the study as intensity increased or remained constant, similar to findings in over-reaching endurance athletes, suggesting disturbances in sleep quality as hard training increased. ${ }^{40}$

There are additional factors that could explain the lack of improvement during the intervention phase. Primary among them was the practice schedule and training plan of the participants. All participants were on the same training plan from the coaches; however it is possible that athletes were doing additional workouts on their own in conjunction with the testing. This could affect how well rested participants were for the test, influencing their performance week to week. It is important to note that this study was performed in a collegiate sports setting, which speaks to its ecological validity.

Another factor that could have contributed to the results we saw was nutrition, as diet can have a large impact on performance. ${ }^{41-42}$ Additionally, lifestyle stress or school stress could have impacted recovery and performance of participants. During the study timeframe there were several exams for classes. Studying would have likely resulted in later nights and higher levels of stress for the participants. We could indirectly monitor stress through the daily HRV recordings for each athlete, although these cannot give specific causes of stress, merely systemic stress and how well the individuals were adapting.

\section{Limitations}

There were several limitations to this study. First, the athletes were unable to achieve the necessary time in bed and time asleep. As stated previously, this is a group with early practice times, a unique population in this research as most sports studied have afternoon or evening practices. ${ }^{7-9}$ Our participants would have to aim for a bedtime of $8 \mathrm{pm}$ each night to meet the nine hours of sleep required for the intervention. Even with napping they did not achieve the prescribed sleep during intervention. Better adherence might be possible if a study was to look at the effects of smaller increases in sleep, such as a set percent increase over baseline or a specific amount of time, or to assess time in bed rather than total sleep.

Naps have been shown to be effective as supplementing lost sleep but there is a possibility that these could have impacted adherence to strict bedtimes at night. Additionally, the sleep tracker used is designed for bedroom use; episodes of sleep that occurred during the day, such as dozing in class or microsleeps would not have been accounted for. A sensitive wrist actigraph would be able to account for and track these instances. 
Second, because these are student athletes with varying class schedules, the participants self-selected the time of day they completed performance tests. This made it challenging to rule out the effect time of day has on the performance. Research suggests that later practice times will lead to improved performance due to circadian rhythm, subjective feelings of more restful sleep, or both. ${ }^{43-45}$

Thirdly, as this was a small participant group and we did not have a control group, it is difficult to make any determinations or conclusions based on the data collected. This was made more challenging with the data that was missing from imperfect adherence of the participants to the protocol. This missing data also decreases the power of our study. A higher study participation would have been ideal.

Finally, the performance tests used, the OR1-Min, RC1-Min, and Interval tests were all created and implemented following best practice guidelines within the sport of rowing. An alternate method would have been to track a specific workout that the head coach already had as part of the weekly practice schedule instead of implementing something additional to the training program.

Future research should include monitoring of training load and volume during the study, to control for and identify the influence of outside stressors. Non-fatiguing performance tests, such as peak power testing or vertical jump testing will be better for measuring rapid, explosive power. Additionally, tracking a consistent prescribed weekly workout of longer duration would address not having a longer duration test as part of the testing protocol. Finally, using an activity monitor would allow for greater flexibility with napping locations and recording microsleeps, in addition to providing a better gauge of total physical activity on a day-to-day basis for subjects.

\section{Conclusions}

We were unable to evaluate the impact of sleep on performance since athletes did not change their sleep during the intervention. The average sleep time for participants was less than what is recommended by the National Sleep Foundation. Despite the amount of training done, participants did not improve on any of the performance measures used. It is the author's opinion that, in sleeping less than the recommended seven to nine hours, participants hurt their recovery and continued performance over the course of the study. Coaches should include sleep education and reminders as part of their daily interactions with athletes, emphasizing the importance of a good night's sleep on their performance.

\section{Acknowledgments}

Thank you to Dr. Rosney for providing guidance and working with me through this writing process. I am sure on more than one occasion there was cause to pull some hair out but you stuck with me and I really appreciate it.

Thank you to Dr. Wu for consulting on the statistics for this study. 


\section{References}

1. Azboy O, Kaygisiz Z. Effects of sleep deprivation on cardiorespiratory functions of the runners and volleyball players during rest and exercise. Acta Physiologica Hungarica. 2009;96(1):29-36.

2. Martin BJ. Effect of sleep-deprivation on tolerance of prolonged exercise. European Journal of Applied Physiology and Occupational Physiology. 1981;47(4):345-354.

3. Oliver SJ, Costa RJS, Laing SJ, Bilzon JLJ, Walsh NP. One night of sleep deprivation decreases treadmill endurance performance. European Journal of Applied Physiology. 2009;107(2):155-161.

4. Racinais S, Hue O, Blonc S, Le Gallais D. Effect of sleep deprivation on shuttle run score in middle-aged amateur athletes - Influence of initial score. Journal of Sports Medicine and Physical Fitness. 2004;44(3):246-248.

5. Souissi N, Sesboue B, Gauthier A, Larue J, Davenne D. Effects of one night's sleep deprivation on anaerobic performance the following day. European Journal of Applied Physiology. 2003;89(34):359-366.

6. Skein M, Duffield R, Edge J, Short MJ, Mundel T. Intermittent-sprint performance and muscle glycogen after $30 \mathrm{~h}$ of sleep deprivation. Medicine and Science in Sports and Exercise. 2011;43(7):13011311.

7. Mah CD, Mah KE, Kezirian EJ, Dement WC. The effects of sleep extension on the athletic performance of collegiate basketball players. Sleep. 2011;34(7):943-950.

8. Schwartz J, Simon RD, Jr. Sleep extension improves serving accuracy: A study with college varsity tennis players. Physiology \& Behavior. 2015;151:541-544.

9. Swinbourne R, Miller J, Smart D, Dulson DK, Gill N. The effects of sleep extension on sleep, performance, immunity and physical stress in rugby players. Sports. 2018;6(2):42.

10. Javaloyes A, Sarabia JM, Lamberts RP, Moya-Ramon M. Training prescription guided by heartrate variability in cycling. International Journal of Sports Physiology and Performance. 2019;14(1):23-32.

11. Elsenbruch S, Harnish MJ, Orr WC. Heart rate variability during waking and sleep in healthy males and females. Sleep. 1999;22(8):1067-1071.

12. Bosquet L, Papelier Y, Leger L, Legros P. Night heart rate variability during overtraining in male endurance athletes. Journal of Sports Medicine and Physical Fitness. 2003;43(4):506-512.

13. Chatlapalli S, Nazeran H, Melarkod V, et al. Accurate derivation of heart rate variability signal for detection of sleep disordered breathing in children. Proceedings of the 26th Annual International Conference of the Ieee Engineering in Medicine and Biology Society. 2004;1:538-541.

14. Hynynen E, Uusitalo A, Konttinen N, Rusko H. Heart rate variability during night sleep and after awakening in overtrained athletes. Medicine and Science in Sports and Exercise. 2006;38(2):313317.

15. Hynynen E, Vesterinen V, Rusko H, Nummela A. Effects of Moderate and Heavy Endurance Exercise on Nocturnal HRV. International Journal of Sports Medicine. 2010;31(6):428-432.

16. Saboul D, Balducci P, Millet G, Pialoux V, Hautier C. A pilot study on quantification of training load: The use of HRV in training practice. European Journal of Sport Science. 2016;16(2):172-181.

17. Plews DJ, Laursen PB, Stanley J, Kilding AE, Buchheit M. Training adaptation and heart rate variability in elite endurance athletes: Opening the door to effective monitoring. Sports Medicine. 2013;43(9):773-781.

18. Flatt AA, Esco MR, Nakamura FY, Plews DJ. Interpreting daily heart rate variability changes in collegiate female soccer players. Journal of Sports Medicine and Physical Fitness. 2017;57(6):907-915.

19. Plews DJ, Laursen PB, Buchheit M. Day-to-day heart-rate variability recordings in worldchampion rowers: Appreciating unique athlete characteristics. International Journal of Sports Physiology and Performance. 2017;12(5):697-703.

20. Koelling S, Steinacker JM, Endler S, Ferrauti A, Meyer T, Kellmann M. The longer the better: Sleep-wake patterns during preparation of the World Rowing Junior Championships. Chronobiology International. 2016;33(1):73-84. 
21. Chung F, Abdullah HR, Liao P. STOP-Bang Questionnaire: A practical approach to screen for obstructive sleep apnea. Chest. 2016;149(3):631-638.

22. Johns MW. A new method for measuring daytime sleepiness - the epworth sleepiness scale. Sleep. 1991;14(6):540-545.

23. Buysse DJ, Reynolds CF, Monk TH, Berman SR, Kupfer DJ. The Pittsburgh Sleep Quality Index - a new instrument for psychiatric practice and research. Psychiatry Research. 1989;28(2):193-213.

24. Weinreich G, Terjung S, Wang Y, Werther S, Zaffaroni A, Teschler H. Validation of a noncontact screening device for the combination of sleep-disordered breathing and periodic limb movements in sleep. Sleep and Breathing. 2018;22(1):131-138.

25. Tal A, Shinar Z, Shaki D, Codish S, Goldbart A. Validation of contact-free sleep monitoring device with comparison to polysomnography. Journal of Clinical Sleep Medicine. 2017;13(3):517-522.

26. Altini MaA, O. HRV4Training: Large-scale longitudinal training load analysis in unconstrained free-living settings using a smartphone application. $38^{\text {th }}$ Annual International Conference of the IEEE Engineering in Medicine and Biology Society (EMBC). 2016:2610-2613.

27. Altini $\mathrm{MaVH}, \mathrm{C}$. Relation between estimated cardiorespiratory fitness and running performance in free-living: An analysis of hrv4training data. 2017 IEEE EMBS International Conference on Biomedical \& Health Informatics. 2017:249-252.

28. Tibana RA, de Sousa NMF, Prestes J, Feito Y, Ernesto C, Voltarelli FA. Monitoring training load, well-being, heart rate variability, and competitive performance of a functional-fitness female athlete: A case study. Sports. 2019;7(2):35.

29. Plews DJ, Scott B, Altini M, Wood M, Kilding AE, Laursen PB. Comparison of heart-ratevariability recording with smartphone photoplethysmography, Polar H7 chest strap, and electrocardiography. International Journal of Sports Physiology and Performance. 2017;12(10):1324-1328.

30. Plews DJ, Laursen PB, Le Meur Y, Hausswirth C, Kilding AE, Buchheit M. Monitoring training with heart-rate variability: How much compliance is needed for valid assessment? International Journal of Sports Physiology and Performance. 2014;9(5):783-790.

31. Ayar S, Karacabey K, Aksu A, Ayar A. Comparison of perceived and objectively measured sleep quality among elite rowing athletes. International Journal of Applied Exercise Physiology. 2019;8.

32. Williams S, Booton T, Watson M, Rowland D, Altini M. Heart rate variability is a moderating factor in the workload-injury relationship of competitive CrossFit (TM) athletes. Journal of Sports Science and Medicine. 2017;16(4):443-449.

33. Malik M., \& Camm, A.J.Heart rate variability: Electrophysiology, pacing, and arrhythmia. Clinical Cardiology. 1990;13(8): 570-576.

34. Pichot V, Roche F, Gaspoz JM, et al. Relation between heart rate variability and training load in middle-distance runners. Medicine and Science in Sports and Exercise. 2000;32(10):1729-1736.

35. Kamdar BB, Kaplan KA, Kezirian EJ, Dement WC. The impact of extended sleep on daytime alertness, vigilance, and mood. Sleep Medicine. 2004;5(5):441-448.

36. Simon RD, Schwartz J. Sleep extension improves college athletic performance. Sleep. 2005;28:A130-A130.

37. O’Donnell S\& Driller MW. Sleep-hygiene Education improves Sleep Indices in Elite Female Athletes.International Journal of Exercise Science. 2017; 10(4): 522.

38. Swinbourne R, Gill N, Vaile J, Smart D. Prevalence of poor sleep quality, sleepiness and obstructive sleep apnoea risk factors in athletes. European Journal of Sport Science. 2016;16(7):850858.

39. Driller MW, Lastella M, Sharp AP. Individualized sleep education improves subjective and objective sleep indices in elite cricket athletes: A pilot study. Journal of Sports Sciences. 2019;37(17):2021-2025.

40. Hausswirth C, Louis J, Aubry A, Bonnet G, Duffield R, Le Meur Y. Evidence of disturbed sleep and increased illness in overreached endurance athletes. Medicine and Science in Sports and Exercise. 2014;46(5):1036-1045. 
41. Burke LM. Nutrition and athletic performance (vol 48, pg 543, 2016). Medicine and Science in Sports and Exercise. 2017;49(1):222-222.

42. Thomas DT. Position of the Academy of Nutrition and Dietetics, Dietitians of Canada, and the American College of Sports Medicine: Nutrition and Athletic Performance. Journal of the Academy of Nutrition and Dietetics. 2016;116(3):501-528.

43. Deschodt VJ, Arsac LM. Morning vs. evening maximal cycle power and technical swimming ability. Journal of Strength and Conditioning Research. 2004;18(1):149-154.

44. Grant MC, Glen J. An investigation into sleep patterns and the effect of time of day on performance in youth swimmers. Biological Rhythm Research. 2018;49(6):830-843.

45. Heishman AD, Curtis MA, Saliba E, Hornett RJ, Malin SK, Weltman AL. Comparing performance during morning vs. afternoon training sessions in intercollegiate basketball players. Journal of Strength and Conditioning Research. 2017;31(6):1557.

\section{ORCID IDs}

Thaddeus Babiec: https://orcid.org/0000-0002-3101-8428 